\title{
Comparison of pain, cortisol levels, and psychological distress in women undergoing surgical termination of pregnancy under local anaesthesia versus intravenous sedation Sharain Suliman*1, Todd Ericksen ${ }^{1}$, Peter Labuschgne ${ }^{2}$, Renee de Wit ${ }^{1}$, Dan J Stein ${ }^{1}$ and Soraya Seedat ${ }^{1}$
}

Address: ${ }^{1}$ MRC Research Unit on Anxiety and Stress Disorders, Department of Psychiatry, University of Stellenbosch, Tygerberg, Cape Town, 7505 , South Africa and ${ }^{2}$ Marie Stopes Clinic, Cape Town, 8001, South Africa

Email: Sharain Suliman* - sharain@sun.ac.za; Todd Ericksen - todd@ericksenfamily.com; Peter Labuschgne - pwl@sedation.co.za; Renee de Wit - rdewit@cashette.com; Dan J Stein -djs2@sun.ac.za; Soraya Seedat - sseedat@sun.ac.za

* Corresponding author

Published: 12 June 2007

BMC Psychiatry 2007, 7:24 doi:10.1186/147/-244X-7-24
Received: 23 October 2006

Accepted: 12 June 2007

This article is available from: http://www.biomedcentral.com/I47I-244X/7/24

(C) 2007 Suliman et al; licensee BioMed Central Ltd.

This is an Open Access article distributed under the terms of the Creative Commons Attribution License (http://creativecommons.org/licenses/by/2.0), which permits unrestricted use, distribution, and reproduction in any medium, provided the original work is properly cited.

\begin{abstract}
Background: The weight of evidence suggests that women who freely choose to terminate a pregnancy are unlikely to experience significant mental health risks, however some studies have documented psychological distress in the form of posttraumatic stress disorder and depression in the aftermath of termination. Choice of anaesthetic has been suggested as a determinant of outcome. This study compared the effects of local anaesthesia and intravenous sedation, administered for elective surgical termination, on outcomes of pain, cortisol, and psychological distress.
\end{abstract}

Methods: 155 women were recruited from a private abortion clinic and state hospital (mean age: $25.4 \pm 6.1$ years) and assessed on various symptom domains, using both clinician-administered interviews and self-report measures just prior to termination, immediately post-procedure, and at I month and 3 months post-procedure. Morning salivary cortisol assays were collected prior to anaesthesia and termination.

Results: The group who received local anaesthetic demonstrated higher baseline cortisol levels (mean $=4.7$ vs 0.2 ), more dissociative symptoms immediately post-termination (mean $=14.7 \mathrm{vs}$ 7.3), and higher levels of pain before (mean $=4.9 \mathrm{vs} 3.0$ ) and during the procedure (mean $=8.0 \mathrm{vs}$ 4.4). However, in the longer-term ( $I$ and 3 months), there were no significant differences in pain, psychological outcomes (PTSD, depression, self-esteem, state anxiety), or disability between the groups. More than $65 \%$ of the variance in PTSD symptoms at 3 months could be explained by baseline PTSD symptom severity and disability, and post-termination dissociative symptoms. Of interest was the finding that pre-procedural cortisol levels were positively correlated with PTSD symptoms at both I and 3 months.

Conclusion: High rates of PTSD characterise women who have undergone surgical abortions (almost one fifth of the sample meet criteria for PTSD), with women who receive local anaesthetic experiencing more severe acute reactions. The choice of anesthetic, however, does not appear to impact on longer-term psychiatric outcomes or functional status. 


\section{Background}

Despite the high rate of elective abortions, controversy still exists about the psychological risks associated with this procedure. On the one hand, while the weight of evidence suggests that when women freely choose to terminate a pregnancy they are unlikely to experience significant mental health risks [1-3], some studies have documented psychiatric disorders such as posttraumatic stress disorder (PTSD) in the aftermath of termination [46]. Several factors have been associated with post-termination responses, including type of abortion procedure and age. With regards to age some studies have found that younger age may be associated with a higher risk of negative responses post-abortion $[7,8]$, while others that have compared psychological responses in adolescents under 18 years of age with those over 18 have shown no differences [2].

Notably, no significant differences in anxiety or depressive symptoms have been documented in women undergoing medical or surgical termination $[9,10]$. However, in women who undergo surgical termination, awareness during anaesthesia especially if it is associated with pain, [11] may be so traumatic so as to place some women at risk of developing PTSD [12]. Seeing the foetus may be traumatic [7], while awareness or consciousness during experiences of blood, pain and death (of the foetus) have also been associated with PTSD-type symptoms [10], namely re-experiencing (cycle of intrusion and denial), avoidance (numbing) and perception of the termination as traumatic [6].

Several studies, mostly retrospective, have described the development of PTSD in patients who experience awareness under anaesthesia for various surgical procedures [13-15]. However, findings have not been altogether consistent. For instance, Slade et al. [10] documented more postabortion distress (including nightmares, flashbacks, and unwanted thoughts related to the procedure) in women who received general anaesthesia (GA) (with presumably no or little intraoperative awareness) compared with women who received local anaesthesia (LA).

The trend to perform surgical abortions under LA rather than GA is growing, with many clinics now providing supplementary medication in the form of conscious/intravenous sedation (IS) (i.e., opioid analgesia) in an effort to improve pain control. However, the benefit of IS in women undergoing abortions is unclear. It has been noted that women who receive IS report less subjective distress and pain than those who receive LA $[16,17]$. On the other hand, it has been suggested that IS improves patient satisfaction but not pain $[18,19]$. For example, Rawling \& Wiebe [20] and Wiebe [21] found no or questionably significant relief in women who received IS.
In view of the lack of prospective studies on introperative and postoperative effects of pain and awareness on longterm psychological, social and physical well-being, this study was conducted to examine the effects of elective surgical pregnancy termination on mental and physical health status when IS versus traditional LA techniques are used.

We aimed to determine if either LA or IS predicted posttraumatic stress disorder, depression, anxiety or physical ill health in women at 1-month and 3-month follow-up, and to examine other predictors (e.g. pain, self-esteem, age of woman, gestational age, cortisol), if any, of postabortion PTSD at 3 months. We hypothesized that women who received IS would have significantly less pain, PTSD, depression and anxiety symptoms compared with women who received no sedation. Based on previous findings, we also expected baseline cortisol levels to be inversely correlated with PTSD at 3 months. PTSD is known to be characterized endocrinologically by an alteration of hypothalamic-pituitary-adrenocortical (HPA) axis function with lowered cortisol secretion [22]. There is evidence, too, for a longitudinal inverse correlation between PTSD symptoms and morning salivary cortisol levels [23], although this has not been well characterized.

\section{Methods \\ Subjects}

155 consecutive referrals ( 80 from a private abortion clinic in Cape Town and 75 from a state-funded secondary level general hospital with an obstetrics/gynaecology unit) were recruited. Referrals were women undergoing surgical termination of pregnancy under local anaesthesia or sedation. Both sites offered local anaesthesia and sedation and the choice of anaesthetic was determined by patient preference. To be eligible, women had to be 16 years of age and older, be at 6-20 weeks of gestation, have an unintended pregnancy that was not the result of rape, and be fluent in English. Women were invited to participate at the time they received counselling for their termination.

The mean age of participants was 25.4 years (range: 16 41 years; SD: 6.1 years), with $34 \%$ of women in the 16 to 21 year age group. Age differences were evident between the anaesthetic groups, with women in the IS group significantly older than women in the LA group $(27.5 \pm 6.6$ years vs. $24.2 \pm 5.4$ years, $t=3.2, p=0.002)$.

There were no significant group differences with respect to ethnicity, marital status, number of children, previous miscarriages, previous abortions, or previous assault history. Most women were of mixed ethnicity [Coloured] (48.3\%) and were living with a partner (55.6\%). 51.7\% of participants had one or more living children. $59.0 \%$ had 
had an abortion prior to this one and $12.6 \%$ had previously miscarried. Approximately $42 \%$ of women had experienced some type of assault in their lives, with the most common forms of assault being mugging (10.6\%), rape $(9.3 \%)$ and domestic violence $(9.3 \%)$.

\section{Procedure}

This study was conducted with the approval of the Committee for Human Subjects at the University of Stellenbosch, and was in compliance with the Helsinki Declaration. Written, informed consent was obtained from all subjects prior to enrolment. Participants were assured that their responses would be confidential and anonymous, and that refusal to participate would in no way jeopardise their management. Eligible women were interviewed at 4 time points. A pre-termination assessment (T1) was conducted approximately $1-3$ hours prior to the procedure and prior to administration of anaesthetic. The termination procedure followed (this varied in duration from 5 to 35 minutes). Follow-up questionnaires were then completed in the recovery room just prior to discharge (T2), then at 1 month (T3) and at 3 months (T4) post-procedure. All assessments were done in person, except for the 3-month assessment (T4) which was telephonic. A Demographic Questionnaire was devised for the study and included information on age, ethnicity, religion, marital status, education, socio-economic level, prior medical/surgical/psychiatric history, prior live births, prior abortions. The research psychologist who conducted the assessments was blind to the anaesthetic status of subjects.

\section{Anaesthesia}

The local anaesthetic used at both centres was Petercaine $1 \%$ injection (i.e. $10 \mathrm{mg}$ per $\mathrm{ml}$ of lignocaine hydrochloride monohydrate). In addition, women at the state hospital received pre-medication comprising indomethacin (100 mg suppository) and diazepam (10 mg orally). The sedation was done using one of two combinations: Propofol 1\% (20 ml) + Alfentanyl $1 \mathrm{mg}(2 \mathrm{ml})+$ Ketamine 20 $\mathrm{mg}(2 \mathrm{ml})$ or Propofol $1 \%(20 \mathrm{ml})+$ Ramifentanyl, administered intravenously. The induction dose was 0.85 mg per kg (Propofol) and increased as necessary to achieve the desired effect. Duration of administration varied according to patient needs and duration of procedure. All patients (local anaesthetic and sedation) received Misoprostol $(400 \mathrm{mg} / 800 \mathrm{mg} / 1200 \mathrm{mg}$ ) and those undergoing sedation also received Midazolam $7.5 \mathrm{mg} \pm 2$ hours orally pre-op. Use of these methods was dependent on the locale of the procedure, approaches of the providers, wishes of, and affordability to the participant.

\section{Instruments}

The following were administered at the pre-termination assessment (T1):

\section{Visual Analogue Scale [VAS] for pain}

The VAS consists of a $10 \mathrm{~cm}$ line with the endpoints 0 ('no pain') and 10 ('worst pain').

Participants are asked to indicate their current pain intensity on the scale using a cross (X). The VAS is a widely used measure of pain intensity and has also been shown to have adequate sensitivity to change in pain associated with treatment across many populations and settings [24].

\section{Clinician-Administered PTSD Scale [CAPS-I]}

The CAPS [25] has become the 'gold' standard for outcome studies in PTSD. It provides current and lifetime PTSD diagnostic information in addition to frequency $(0$ $=$ never; $4=$ daily or almost every day $)$ and severity $(0=$ none; $4=$ extreme) scores of individual PTSD symptoms. Participants are asked to respond to the questions with reference to the previous week and with a particular trauma in mind. participants are asked to respond to the questions with reference to the last week and a particular trauma in mind at $\mathrm{T} 1$, and with reference to the termination as the index trauma at $\mathrm{T} 3$ and $\mathrm{T} 4$.

The recommended score of $\geq 50$ was used to identify women with PTSD.

\section{Davidson Trauma Scale [DTS]-self report}

The DTS [26] is self-rated measure of PTSD comprising 17 items that correspond to each of the DSM-IV symptoms of PTSD. The respondent rates frequency $(0=$ never; $4=$ daily or almost every day) and severity ( $0=$ none; $4=$ extreme $)$ for each item on a 5-point scale. A cut-off score of 40 on the DTS is considered to be indicative of PTSD. As with the CAPS, participants are asked to respond to the questions with reference to the last week and a particular trauma in mind at $\mathrm{T} 1$, and with reference to the termination at $\mathrm{T} 3$ and $\mathrm{T} 4$.

\section{Spielberger State-Trait Anxiety Inventory [STAI] state scale}

The STAI [27] is a self-rated scale that measures current anxiety symptoms on a scale of 1 to 4 ( 1 = almost never; 4 = almost always). A score of $\geq 40$ is considered 'high anxiety'. The State version of the STAI was used.

\section{Beck Depression Inventory}

The Beck Depression Inventory (BDI) [28] is a self-rated measure of depressive symptoms. Respondents choose from 4 possible answers ranging in intensity from 0 to 3 . As a screening test for depression, recommended cut-off points are: 0-9 non-depressed, 10-15 mild depression; 16-23 moderate depression; and $\geq 24$ severe depression.

\section{Rosenberg Self-Esteem Inventory}

The Rosenberg Self-Esteem Inventory (RSI) is a well-validated and widely used measure of self-esteem [29]. 
Respondents indicate how they feel on scales of 1 (strongly agree) to 4 (strongly disagree). A higher score is indicative of a better sense of self-worth. The range of possible scores is $10-40$, with mean scores below 20 indicating average responses on the negative side of self-esteem, and mean scores above 30 indicating average responses on the positive side of self-esteem [30].

\section{Schneier Disability Scale (SDS)}

The SDS assesses current and lifetime impairment in 8 domains of functioning and each item is rated separately for current and most severe lifetime disability on a 5point, descriptively anchored scale ranging from 0 (no impairment) to 4 (severe impairment). The item scores may be totaled to obtain 2 summary scores, one rating overall current disability and the other most severe lifetime disability. Alternatively item scores may be considered individually to provide descriptive information on the pattern of impairment across domains.

Immediately post-procedure (T2), the following measures were administered: (i) Visual Analogue Scale (VAS) for Pain and (ii) Dissociative Experiences Scale-Taxon [DES-T]. The DES-T, derived from the 28-item Dissociative Experiences Scale [DES] [31], is an 8-item subscale designed to discriminate pathological from non-pathological forms of dissociation [32,33]. The scale focuses on pathological changes in consciousness, such as dissociative amnesia, depersonalization and derealization. Subjects are asked to allocate a percentage of time that they experience each type of dissociative phenomenon $(0 \%-100 \%)$.

All assessments done pre-termination (except for the demographic and pain assessments) were repeated at the 1-month (T3) \& 3-month (T4) follow-up visits.

Data from 151 participants were included in the final analysis (78 from the private clinic and 73 from the state hospital). Four patients were excluded owing to missing data at $\mathrm{T} 1$ (pre-termination). Of the 151 participants, 76 $\%(\mathrm{~N}=115)$ completed assessments immediately postprocedure, $44 \%(\mathrm{~N}=67)$ returned for the 1-month assessment and $37 \%(\mathrm{~N}=56)$ for the 3-month assessment.

Thirty-seven percent of the sample received intravenous sedation (IS) while 63\% received local anaesthetic (LA).

\section{Salivary cortisol}

Morning (09 h 00) saliva specimens were collected at pretermination (T1). Samples (approximately $5 \mathrm{ml}$ volume) were collected and stored at -20 degrees celsius, until assay. Samples were analysed using a commercial radioimmunoassay.

\section{Ethical issues}

Study clinicians did not participate in counselling, ethical decision-making, or termination procedures and adhered to stringent anonymity and confidentiality requirements. In part, owing to anonymity and confidentiality issues involved in following a termination sample over time, some of the original sample was lost to attrition.

\section{Statistical analyses}

The two groups (LA] vs. IS) were compared on primary outcome measures (VAS for pain, CAPS for PTSD) in addition to other demographic and clinical variables, using chi-square tests (for categorical variables) and student's ttests (for numerical variables). Possible associations between the groups were also analysed, controlling for other variables. Women were also stratified by age (16-21 and $>22$ years), and chi-square and student's t-tests were used to compare responses between the groups. For outcomes measured across time (e.g., PTSD, depression, selfesteem, physical health scores), and in comparing baseline differences of those who dropped out of the study with those who completed it as well as gestational age of women with and without PTSD at 3 months, mean differences between the groups were analysed using student's ttests. Correlations were examined among the different outcomes (e.g. PTSD, depression, self-esteem) using Pearson correlation coefficients. The DTS, BDI, and Spielberger scales were categorized into high and low scores (determined by each scale's recommended cut-off) to determine thefrequency of cases above the cut-off for clinical significance. In order to determine whether or not there were any statistically significant differences between groups over time, we performed GLM Repeated Measures ANOVAs. The influence of demographic/clinical characteristics on PTSD outcome was examined by simultaneously entering variables that emerged as statistically significant from bivariate analyses into a multiple linear regression equation to predict PTSD scores at 3-months. The significant variables were then entered into a stepwise regression which produced 3 models. The semi-partial correlation coefficients and betas were computed in order to evaluate the unique contribution of each predictor variable. For all analyses, statistical significance was set at $p$ $<.05$ and all tests 2-tailed.

\section{Results \\ Differences between LA and IS groups \\ (i) Pain}

Participants who received IS reported less pain, (i.e., significantly lower pain scores) than those who received LA both at baseline (IS: $3.0 \pm 2.3$; LA: $4.9 \pm 3.1 ; p=0.000$ ) and during surgery (IS: $4.4 \pm 3.0$; LA: $8.0 \pm 1.0 ; p=<0.005$ ). 


\section{(ii) PTSD}

No significant differences in PTSD symptom status (CAPS and DTS total scores) were observed between the groups at either the 1 month or 3 month assessments. At baseline, $11.3 \%$ of the sample met criteria for a diagnosis of PTSD on the CAPS (IS: 7.3\%; LA: 14.1\%), 17.5\% met criteria at 1 month (IS: 9.4\%; LA: $28.0 \%$ ), and $18.2 \%$ at 3 months (IS: $10.0 \%$; LA: $28.0 \%$ ). No significant association between group status (IS/LA) and the three month CAPS scores was found.

\section{(iii) Other psychiatric outcomes}

Women who received IS experienced significantly less dissociation immediately following the procedure than women who received LA (IS: $7.3 \pm 11.3$; LA: $14.7 \pm 17.6$; $p$ $=0.01)$. With respect to longer-term outcome, there were no significant differences in depression, self-esteem, state anxiety, and disability scores between IS and LA groups at 1 and 3 months.

With respect to depressive symptoms, at pre-termination $21.9 \%$ of the sample had scores suggestive of clinical depression (IS: 21.8\%; LA: 23.1\%), while at both 1 and 3 months, $20 \%$ of women (IS: $13.8 \%$; LA: $25.7 \%$ ) had scores suggestive of clinical depression. 63.9\% scored 'high' on state anxiety (STAI) at pre-termination (IS: 66.0\%; LA: 62.6\%), while at both 1 and 3 months, 56.3\% of women (IS: 51.7\%; LA: 60.0\%) had 'high' STAI scores.

Secondary analyses were conducted to compare women with and without a PTSD diagnosis on pain and psychiatric symptoms at 1 and 3 months follow-up. Women with a PTSD diagnosis on the CAPS endorsed significantly more pain after the procedure at both 1 month (With PTSD: $7.0 \pm 3.3$; Without PTSD: $3.9 \pm 2.5 ; p=0.049)$, and 3 months (With PTSD: $8.0 \pm 2.4$; Without PTSD: $3.7 \pm 2.4$; $p=0.012)$. They also had higher CAPS, DTS, anxiety, and disability scores at 1 month. Notably, those who met PTSD criteria at 3 months after termination had significantly lower depression (With PTSD: $6.8 \pm 6.7$; Without PTSD: $27.8 \pm 8.2 ; p=0.003$ ) and self-esteem (With PTSD: $23.0 \pm 1.9$; Without PTSD: $31.9 \pm 6.1 ; p=0.000)$ scores at one month than those who did not meet criteria. GLM Repeated Measures ANOVAs for baseline, 1 and 3 month CAPS scores, baseline, 1 and 3 month DTS scores, and baseline, 1 and 3 month BDI scores comparing anaesthetic status were performed, while controlling for age, baseline cortisol, and prior trauma exposure. Results indicated that there was no significant differences between groups (IS/LA) over time. However there were significant differences between pre-termination and 1 month DTS scores $(\mathrm{F}=4.52, \mathrm{df}=4,18, p=0.03)$ and 1 and 3 month BDI scores, $\mathrm{F}=16.40, \mathrm{df}=2,18, p=0.00)$. There were no significant baseline differences between participants who dropped out of the study prior to completion and those who completed it. However, women with PTSD 3 months after termination were further along in their pregnancy than those without PTSD (gestational age: With PTSD: $13.2 \pm 3.3$; Without PTSD: $9.7 \pm 4.2 ; p=0.023)$.

\section{(iv) Age}

At baseline, younger women (16 - 21 years) endorsed more depressive symptoms (Younger: $16.2 \pm 9.1$; Older: $12.7 \pm 9.0 ; p=0.027)$ and had lower self-esteem scores than older women (> 21 years) (Younger: $27.2 \pm 6.0$; Older: $30.3 \pm 5.6 ; p=0.003)$. They also, reported significantly more pain post-termination (Younger: $5.3 \pm 3.1$; Older: $3.7 \pm 2.8 ; p=0.011)$ and had higher anxiety scores at 1 month (Younger: $48.2 \pm 8.9$; Older: $41.9 \pm 11.9 ; p=$ 0.026 ) and 3 months (Younger: $48.7 \pm 9.0$; Older: $41.9 \pm$ $11.9 ; p=0.021)$.

\section{(v) Salivary cortisol}

Women in the IS group had significantly lower baseline cortisol levels than those in the LA group (IS: $0.2 \pm 0.2$; LA: $4.7 \pm 17.6 ; p=<0.001)$. Cortisol levels were positively correlated with baseline pain $(\mathrm{r}=0.5 ; p=0.000)$ and state anxiety $(\mathrm{r}=0.3 ; p=0.006)$ and negatively correlated with baseline self-esteem $(\mathrm{r}=-0.4 ; p=0.009)$. At 1 and 3 months, baseline cortisol correlated positively with total CAPS scores (i.e. higher baseline cortisol levels were associated with more PTSD symptomatology) $(\mathrm{r}=0.3 ; p=$ 0.043 ). Additionally, women with "high" anxiety scores at pre-termination and 1 month after the procedure had higher baseline cortisol levels than those with "low" anxiety scores (High anxiety: $0.5 \pm 0.6$; Low anxiety: $0.3 \pm 0.2$; $p=0.024)$.

In summary, women who received IS reported significantly less pain before and during the procedure, and had less severe dissociation and lower baseline cortisol than women who received LA.

\section{Predictors of PTSD at 3 months}

To determine which variable/s, if any, independently predicted CAPS PTSD scores at 3 months, variables that emerged as statistically significant in bivariate analyses, namely PTSD (CAPS) scores at baseline, disability at baseline (Schneier) and post-termination dissociation (DEST) were entered as predictor variables into a forward stepwise linear regression analysis. This procedure produced three models. The first model retained baseline CAPS scores and accounted for $44.7 \%$ (adjusted $\mathrm{R}^{2}=0.43$ ) of the variance in 3-month CAPS scores. The second model retained CAPS and disability scores at baseline and accounted for $57.1 \%$ (adjusted $\mathrm{R}^{2}=0.54$ ) of the variance in PTSD symptoms. The third model retained CAPS and disability scores at baseline and post-procedural dissociation. This model accounted for $66.1 \%$ (adjusted $\mathrm{R}^{2}=$ 0.62 ) of the variance in PTSD symptoms. The semi-partial 
correlation coefficients and betas were also computed in order to determine the unique contribution of each predictor variable.

\section{Discussion}

Our results do not demonstrate any notable long-term differences in outcome between the two groups (sedation/ LA). Thus, PTSD, depression, state anxiety, and disability were not associated with the type of anaesthesia received. Although not statistically significant, women who underwent local anaesthesia were more likely to meet PTSD criteria.

The CAPS was used to establish a diagnosis of PTSD based on traumatic event exposure prior to termination and, at subsequent assessments, a diagnosis of PTSD secondary to the termination procedure. The point prevalence of PTSD at baseline was $11.3 \%$, which is comparable with US community norms [10] but lower than the rate obtained in another local community study [34]. The prevalence of PTSD after termination was $17.5 \%$ and $18.2 \%$ at one and three months respectively. This is higher than the rate found by Rue et al. [5] in American and Russian women ( $14.3 \%$ and $0.9 \%$ respectively), but lower than that found in other studies of pregnancy loss, for example, by Engelhardt et al. [35]. Of note, PTSD symptom status at 3 months was predicted by baseline (pre-termination) severity of PTSD symptoms and the level of pain experienced, but was not predicted by the presence of other psychopathology (depression, state anxiety, self-esteem, and functional disability. Women with PTSD were more likely to be further along in their pregnancies. This has been noted in studies by Grimes and Cates [36] and Davies et al. [37], and has been explained on the basis that women in an earlier stage of pregnancy have had a shorter relationship with the foetus, are less likely to have experienced foetal movements and, as such, may be at lower risk. In addition, high levels of pre-existing PTSD and functional disability, as well as post-termination dissociation appear to contribution to PTSD in the longer term in this population. Thus it would follow that screening women pre-termination for PTSD and disability and posttermination for high levels of dissociation is important in order to help identify women at risk of PTSD and to provide follow-up care. Both dissociation [38] and pre-existing PTSD $[39,40]$ have been identified as risk factors for PTSD in other trauma populations.

Baseline rates of depression (21.9\% had scores indicative of clinical depression) and general anxiety (63.9\% had high STAI scores) were also high, with prevalence rates similar to those found in other studies. For example, in their review, Bradshaw and Slade [41] observed that prior to termination, levels of 'caseness' on measures of anxiety, depression and overall psychological distress ranged from
15 to $69 \%$. Anxiety scores in the present study are also comparable with those documented in pre-operative samples [42]. However, rates of depression, and state anxiety (as measured on the DTS, BDI and Spielberger, respectively) at post-termination did not differ significantly from pre-termination rates. Rates of depression were similar to population based rates in the US [8] but much lower than found previously in a local sample (Carey et al., 2003) [34]. Similar to the findings reported here, several other studies have found lower levels of depression $[7,43,8])$ and lower state anxiety scores $[44]$ post-abortion compared with pre-abortion.

The finding of lower baseline pain levels in women who received IS is consistent with observations by Wells [16] and Rawlings and Weibe $[17,20]$. At post-termination, the LA group reported higher levels of dissociation than the IS group. It could be argued that higher levels of pain in the LA group might have contributed to a greater propensity to dissociate. For example Duckworth et al. [45] observed that rates of dissociation were generally higher in persons with pain, even among "non-traumatised" patients.

Higher cortisol levels in the local anaesthesia group were consistent with the greater experience of pain. Other studies, for example Zimmer et al. [46], have also shown significant correlations between pain and cortisol levels. Additionally, in this sample higher pre-procedural cortisol levels were positively correlated with PTSD symptoms at 1 month and 3 months. The correlation between cortisol and pain and cortisol and anxiety in this study may further be representative of the association between anxiety and pain as suggested by Belanger, Melzack \& Lauzon [47] and Taenzer [42]. However, whether higher baseline cortisol is an etiologic marker of PTSD symptom severity or is merely an epiphenomenon of some other process remains unclear and warrants further investigation.

Pain intensity has been significantly correlated with subjective distress [16], including stress and anxiety [48] and it has been suggested that the experience of pain during termination cannot be separated from the anxiety and trepidation that are experienced before the procedure [19]. In fact, the level of pre-abortion anxiety has been positively associated with pain experienced during abortion [47] and is consistent with the role of anxiety in pain perception and response [42]. Consistent with findings from previous investigations [16], pain and PTSD symptoms tended to co-occur in the sample.

There are several limitations to this study. First, there was a high rate of attrition over the course of the study leaving a small final sample ( $37 \%$ of the original sample). It might be that participants who were lost to follow-up were lost because of their higher levels of postabortion 
Table I: Comparison of Clinical Outcomes in Women receiving Intravenous Sedation (IS) versus Local Anaesthetic (LA)

\begin{tabular}{|c|c|c|c|c|c|c|c|c|c|}
\hline & & \multicolumn{2}{|c|}{ Pre-termination $(N=15 I)$} & \multicolumn{2}{|c|}{ Post-termination $(\mathrm{N}=1 \mid \mathrm{I})$} & \multicolumn{2}{|c|}{ I - month $(N=67)$} & \multicolumn{2}{|c|}{3 - month $(N=56)$} \\
\hline & & Mean & $p$ & Mean & $p$ & Mean & $p$ & Mean & $p$ \\
\hline \multirow[t]{2}{*}{ PTSD (CAPS) } & IS & $9.2 \pm 22.7$ & 0.355 & N/A & & $16.1 \pm 26.2$ & 0.074 & $17.2 \pm 26.8$ & 0.107 \\
\hline & LA & $12.9 \pm 24.1$ & & & & $29.2 \pm 27.4$ & & $29.2 \pm 27.4$ & \\
\hline \multirow[t]{2}{*}{ PTSD (DTS) } & IS & $42.2 \pm 32.5$ & 0.067 & $N / A$ & & $30.3 \pm 30.4$ & 0.712 & $30.3 \pm 30.4$ & 0.701 \\
\hline & LA & $32.4 \pm 26.2$ & & & & $33.2 \pm 31.1$ & & $33.3 \pm 31.6$ & \\
\hline \multirow[t]{2}{*}{ Depression (BDI) } & IS & $14.5 \pm 9.8$ & 0.602 & N/A & & $10.2 \pm 9.5$ & 0.29 & $10.2 \pm 9.5$ & 0.279 \\
\hline & LA & $13.6 \pm 8.8$ & & & & $13.0 \pm 12.1$ & & $13.1 \pm 12.2$ & \\
\hline \multirow{2}{*}{$\begin{array}{l}\text { Self-esteem } \\
\text { (Rosenberg) }\end{array}$} & IS & $29.8 \pm 6.2$ & 0.331 & N/A & & $30.9 \pm 6.3$ & 0.175 & $30.9 \pm 6.3$ & 0.152 \\
\hline & LA & $28.8 \pm 5.7$ & & & & $28.7 \pm 6.7$ & & $28.5 \pm 6.8$ & \\
\hline \multirow[t]{2}{*}{ Anxiety (Spielberger) } & IS & $44.4 \pm 12.0$ & 0.645 & N/A & & $41.1 \pm 10$ & 0.149 & $41.4 \pm 10.7$ & 0.142 \\
\hline & LA & $43.4 \pm 10.6$ & & & & $45.5 \pm 11.7$ & & $45.6 \pm 11.9$ & \\
\hline \multirow[t]{2}{*}{ Disability (Schneier) } & IS & $4.8 \pm 4.6$ & 0.138 & $\mathrm{~N} / \mathrm{A}$ & & $2.8 \pm 4.0$ & 0.327 & $2.8 \pm 4.0$ & 0.401 \\
\hline & LA & $3.6 \pm 3.2$ & & & & $3.9 \pm 4.3$ & & $3.8 \pm 4.3$ & \\
\hline \multirow[t]{2}{*}{ Cortisol } & IS & $0.2 \pm 0.2$ & 0.001 & $\mathrm{~N} / \mathrm{A}$ & & N/A & & N/A & \\
\hline & LA & $0.5 \pm 0.5$ & & & & & & & \\
\hline \multirow[t]{2}{*}{ Dissociation (DES-T) } & IS & N/A & & $7.3 \pm 11.3$ & 0.01 & $8.1 \pm 13.0$ & 0.142 & $8.1 \pm 13.0$ & 0.213 \\
\hline & LA & & & $14.7 \pm 17.6$ & & $14.4 \pm 20.4$ & & $13.3 \pm 19.7$ & \\
\hline
\end{tabular}

- CAPS: Clinician-Administered PTSD Scale

- DTS: Davidson Trauma Scale

- BDI: Beck Depression Inventory

- DES-T: Dissociative Experiences Scale-Taxon

Table 2: Variables Demonstrating a Significant Association with PTSD Status at 3 months

\begin{tabular}{ccc}
\hline Variable & PTSD & No PTSD \\
\cline { 2 - 3 } & & 3 month mean and SD \\
\hline Baseline PTSD (CAPS) score* & $52.6 \pm 30.7$ & $7.5 \pm 17.8$ \\
Post-abortion pain score* & $8.0 \pm 2.4$ & $3.9 \pm 2.8$ \\
I month PTSD (CAPS) score** & $81.6 \pm 14.3$ & $11.5 \pm 16.9$ \\
I month PTSD (DTS) score** & $93.0 \pm 17.7$ & $20.9 \pm 19.7$ \\
I month Anxiety (STAI) score** & $58.4 \pm 3.3$ & $39.5 \pm 8.6$ \\
I month Depression (BDI) score** & $6.8 \pm 6.7$ & $27.8 \pm 8.2$ \\
I month Self-esteem (RSI) score** & $23.0 \pm 1.9$ & $31.9 \pm 6.1$ \\
I month Disability (SDS)** & $8.8 \pm 2.2$ & $2.89 \pm 4.3$ \\
\hline$*: p<0.05$ & & \\
$* *: p<0.0$ l & &
\end{tabular}

Table 3: Partial Correlations and Standard Beta- values

\begin{tabular}{|c|c|c|c|c|}
\hline & Variables & Standard Beta & Partial Correlation & $P$ \\
\hline Model I & Baseline PTSD (CAPS) Score & 0.668 & 0.668 & 0.000 \\
\hline \multirow{2}{*}{ Model 2} & Baseline PTSD (CAPS) Score & 0.473 & 0.413 & 0.003 \\
\hline & Baseline Disability score & 0.404 & 0.353 & 0.011 \\
\hline \multirow[t]{3}{*}{ Model 3} & Baseline CAPS Score & 0.432 & 0.357 & 0.004 \\
\hline & Baseline Disability Score & 0.389 & 0.339 & 0.007 \\
\hline & Post-termination Dissociation & 0.303 & 0.299 & 0.017 \\
\hline
\end{tabular}


distress (i.e. PTSD and other psychopathology), resulting in lowered rates of psychiatric disorder, although our results indicated no baseline differences between those who dropped out and those who completed the study. Second, salivary cortisol samples, although collected in the morning (within a two hour window) were not all collected at the same time, and there was no control for other variables known to affect cortisol (time of last meal, caffeine consumption, smoking status). Fluctuations in cortisol may have confounded these results and thus should be interpreted very tentatively. Third, this was not a randomised study as the participants were referred to the study only after selecting the type of anaesthetic they would receive. Fourth, sedation was not equally available as a treatment option across both sites and this may have, to some extent, biased these results. For example, the use of ketamine may change the way in which patients remember the procedure. Fifth, the 3-month follow-up was conducted telephonically, contrary to the other 3 assessments, and responses may be partially accounted for by the method of assessment. However, responses at the 1 -month and 3-month assessments remained very similar. Sixth, the majority of participants were of mixed ethnicity (coloured) and while this is the majority ethnic group in the province, these results may not be generalizable to other ethnic groups.

\section{Conclusion}

High rates of PTSD characterise women who have undergone voluntary pregnancy termination. The type of anaesthetic for the termination procedure does not seem to impact on long-term outcomes, however women who receive IS appear to fare better on pain and post-termination dissociative phenomena.

Presently the weight of evidence suggests that abortion does not cause lasting negative consequences $[1,49]$. Nevertheless, longitudinal assessment of multiple variables (e.g., pain, type of termination, anaesthetic procedure, gestational age, cortisol, trauma-related symptoms and other psychophysiological markers) that may potentially impact on subsequent PTSD and major depression is deserving of further study.

\section{Abbreviations}

Beck Depression Inventory: BDI; Clinician Administered PTSD Scale: CAPS; Davidson Trauma Scale: DTS; Dissociative Experiences Scale- Taxon: DES-T; General Anaesthesia: GA; Hypothalmic-pituitary-adrenocortical: HPA; Intravenous Sedation: IS; Local Anaesthesis: LA; Posttraumatic Stress Disorder: PTSD; Rosenberg Self-Esteem Inventory: RSI; Scheneier Disability Scale: SDS; Spielberger State-Trait Anxiety Inventory: STAI; Visual Analogue Scale: VAS

\section{Competing interests}

The author(s) declare that they have no competing interests.

\section{Authors' contributions}

$S$ Suliman drafted the manuscript and participated in the design, co-ordination and data collection of the study. TE helped to draft the protocol and participated in its design and data collection. PL conceived of the study. RDW participated in data collection. DJS helped to draft the manuscript. S Seedat drafted the protocol, designed the study, performed the statistical analysis and helped to draft the manuscript. All authors have read and approved the final manuscript.

\section{Acknowledgements}

This work was supported by the MRC Unit on Anxiety and Stress Disorders and by a Harry Crossley grant. The authors are grateful for the assistance with recruitment that was provided by nursing staff at both Marie Stopes and Karl Bremer clinics and to Professor D Nel (Director, Centre for Statistical Consultation, University of Stellenbosch) and D Fincham (Research Psychologist, MRC Anxiety and Stress Disorders Unit, University of Stellenbosch) for assistance with statistical analyses.

\section{References}

I. Adler N, David H, Major B, Roth SH, Russo NF, Wyatt GE: Psychological factors in abortion. Am Psychol 1992, 47: I I 94- 1204.

2. Pope LM, Adler NE, Tschann JM: Postabortion psychological adjustment: Are minors at increased risk? J Ado Health 200I, 29:2-II.

3. Hess RF: Dimensions of women's long-term post-abortion experience. Am J Mat Chil Nurs 2004, 29:193-198.

4. Barnard C: The long-term psychological effects of abortion. N.H: Institute for Pregnancy Loss. Portsmouth; 1990.

5. Rue VM, Coleman PK, Rue JJ, Reardon DC: Induced abortion and traumatic stress: a preliminary comparison of American and Russian women. Med Sci Monit 2004, I0(10):SR5-16.

6. Speckhard A, Rue M: Postabortion syndrome: an emerging public health concern. J Soc Issues 1992, 48:95-120.

7. Urquart DR, Templeton AA: Psychiatric morbidity and acceptability following medical and surgical methods of induced abortion. BrJ Obstet Gynaecol I99I, 98:396-399.

8. Major B, Cozzarelli C, Cooper ML, Zubek J, Richards C, Wilhite M, Gramzow RH: Psychological responses of women after firsttrimester abortion. Arch Gen Psychiatry 2000, 57(8):777-784.

9. Howie FL, Henshaw RC, Naji SA, Russel IT, Templeton AA: Medical abortion or vacuum aspiration? Two year follow-up of patient preference trial. BrJ Obstet Gynaecol 1997, 104:829-833.

10. Slade P, Heke S, Fletcher J, Stewart P: a comparison of medical and surgical termination of pregnancy: choice, psychological consequences and satisfaction with care. Br J Obstet Gynaecol 1998, I05(I 2): I 288-1295.

II. Asmundson GJG, Wright KD, Stein MB: Pain and PTSD symptoms in female veterans. Euro J Pain 2004, 8:345-350.

12. Osterman JE, van der Kolk BA: Awareness during anesthesia and posttraumatic stress disorder. Gen Hosp Psych 1998, 20:274-28I.

13. Schwender D, Kunze-Kronawitter H, Dietrich P, Klasing D, Frost H, Madler C: Conscious awareness during general anaesthesia: patients' perceptions, emotions, cognition and reactions. $\mathrm{Br}$ J Anaesthesiol 1998, 80:133-139.

14. Osterman JE, Hopper J, Heran WJ, Keane TM, van der Kolk BA: Awareness under anaesthesia and the development of posttraumatic stress disorder. Gen Hosp Psych 200I, 23:198-204.

15. Sebel PS, Bowdle TA, Ghoneim MM, Rampil IJ, Padilla RE, Gan TJ, Domino KB: The incidence of awareness during anesthesia: a multicenter United States study. Anesth Anag 2004, 99:833-839.

16. Wells N: Reducing distress during abortion: A test of sensory information. J Ad Nurs 1992, 17:1050-1056. 
17. Rawling MJ, Wiebe ER: Pain control in abortion clinics. Int J Gynaecol Obstet 1998, 60(3):293-295.

18. Wong $\mathrm{CY}, \mathrm{Ng}$ EH, Ngai SW, Ho PC: A randomised, double blind, placebo-controlled study to investigate the use of conscious sedation in conjunction with paracervical block for reducing pain in termination of first trimester pregnancy by suction evacuation. Hum Rep 2002, 17:1222-1225.

19. Keder LM: Best practices in surgical abortion. Am J Obstet Gynecol 2003, I 89:4I8-422.

20. Rawling MJ, Wiebe ER: A randomized controlled trial of fentanyl for abortion pain. Am J Obstet Gynaecol 200I, I 85(I): I03-107.

21. Weibe ER: Pain control in medical abortion. Int J Gynaecol Obstet 200I, 74:275-280.

22. Yehuda R, Boisoneau D, Lowy MT, Giller EL: Dose-response changes in plasma cortisol and lymphocyte glucocorticoid receptors following dexamethasone administration in combat veterans with and without posttraumatic stress disorder. Arch Gen Psych 1995, 52:583-593.

23. Kellner M, Yehuda R, Arlt J, Weidemann K: Longitudinal course of salivary cortisol in post-traumatic stress disorder. Acta Psychiatr Scand 2002, 105:153-156.

24. Jensen MP, Karoly P: Self-report scales and procedures for assessing pain in adults. In Handbook of pain assessment 2 nd edition. Edited by: Turk DC, Melzack R. New York, NY: Guilford Press; 200I:15-34.

25. Blake DD, Weathers FW, Nagy LM, Kaloupek DG, Gusman FD, Charney DS, Keane TM: The development of a clinician-administered PTSD Scale. J Trauma Stress 1995, 8:75-90.

26. Davidson JR, Book SW, Colket JT, Tupler LA, Roth S, David D, Hertzberg M, Mellman T, Beckham JC, Smith RD, Davidson RM, Katz R, Feldman ME: Assessment of a new self-rating scale for posttraumatic stress disorder. Psychol Med 1997, 27:153-160.

27. Spielberger CD, Goruch RL, Lushene R, Vagg PR, Jacobs GA: Manual for the State-Trait Anxiety Inventory. Palo Alto, CA: Consulting Psychologists Press; 1983.

28. Beck AT, Steer RA: Beck Depression Inventory Manual. San Antonio, TX: Psychological Corporation; 1987.

29. Rosenberg M: Society and the Adolescent Self-Image. Princeton, NJ: Princeton University Press; 1965

30. Russo NF, Zierk KL: Abortion, Child bearing and Women's Well-Being. Profess Psych: Resear and Pract 1992, 4:269-280.

31. Bernstein EM, Putnam FW: Development, reliability, and validity of a dissociation scale. J Nerv Ment Dis 1986, 134:727-735.

32. Waller NG, Putman FW, Carlson EB: Types of dissociation and dissociative types: A taxometric analysis of dissociative experiences. Psychol Methods 1996, I:300-321.

33. Waller NG, Ross CA: The prevalence and biometric structure of pathological dissociation in the general population: taxometric and behaviour genetic findings. J Abnorm Psychol 1997, 106:499-5I0.

34. Carey PD, Stein DJ, Zungu-Dirwayi N, Seedat S: Trauma and Posttraumatic Stress Disorder in an urban Xhosa primary care population: prevalence, comorbidity, and service use patterns. J Nerv Ment Dis 2003, 191:230-236.

35. Engelhardt IM, van den Hout MA, Arntz A: Posttraumatic stress disorder after pregnancy loss. Gen Hosp Psych 200I, 23:62-66.

36. Grimes PA, Cates W: Complications from legally induced abortion. Obstet Gynecol Surv 1995, 34: I77-I9I.

37. Davies V, Gledhill J, McFadyen A, Whitlow B, Economides D: Psychological Outcome in women undergoing termination of pregnancy for ultrasound-detected fetal anomaly in the first and second trimesters: a pilot study. Ultrasound Obstet Gynecol 2005, 25:389-392

38. Breh $\mathrm{DC}$, Seidler $\mathrm{GH}$ : Is peritraumatic dissociation a risk factor for PTSD? J Trauma Dissociation 2007, 8:53-69.

39. Johnson $H$, Thompson $A$ : The development and maintenance of post-traumatic stress disorder (PTSD) in civilian adult survivors of war trauma and torture: A review. Clin Psychol Rev 2007 in press.

40. Copeland WE, Keeler G, Angold A, Costello EJ: Traumatic events and posttraumatic stress in childhood. Arch Gen Psychiatry 2007. 64:577-84

4I. Bradshaw Z, Slade P: The effects of induced abortion on emotional experiences and relationships: a critical review of the literature. Clin Psych Rev 2003, 23:929-958.
42. Taenzer P: Postoperative pain: relationship among measures of pain, mood and narcotic requirement. In Pain, Measurement and Assessment Edited by: Melzak R. Raven, New York; 1983: I I - I 18.

43. Major B, Cozzarelli C: Psychosocial predictors of adjustment to abortion. J Soc Issues 1992, 48:121-142.

44. Cohen L, Roth S: Coping with abortion. J Hum Stress 1984, I0: $140-145$

45. Duckworth, et al.: Dissociation, coping, and adjustment among persons with chronic pain. J Pain 2004 2002, 3:SI32.

46. Zimmer, et al.: Sex, gender and pain. Edited by: Fillingham RB. Seattle: IASP Press; 2000.

47. Belanger E, Melzack R, Lauson P: Pain of first-trimester abortion: a study of psychosocial and medical predictors. Pain 1989 , 36:339-350.

48. Rollman GB: Sex makes a difference: experimental and clinical pain responses. Clin J Pain 2003, 19:204-207.

49. Haris A: Supportive counselling before and after elective pregnancy termination. J Midwif Wom Health 2004, 49: I05-I I 2.

\section{Pre-publication history}

The pre-publication history for this paper can be accessed here:

http://www.biomedcentral.com/1471-244X/7/24/pre pub
Publish with Bio Med Central and every scientist can read your work free of charge

"BioMed Central will be the most significant development for disseminating the results of biomedical research in our lifetime. "

Sir Paul Nurse, Cancer Research UK

Your research papers will be:

- available free of charge to the entire biomedical community

- peer reviewed and published immediately upon acceptance

- cited in PubMed and archived on PubMed Central

- yours - you keep the copyright
BioMedcentral 